\title{
Compliance of a rigid joint of a steel column of I- section on a rigid foundation
}

\author{
Pavel Morozovskiy ${ }^{1,}{ }^{*}$, Egor Kanaev ${ }^{1}$, Nikita Kryzhanovskii ${ }^{1}$ and Rafik Al Malul ${ }^{2}$ \\ ${ }^{1}$ Peter the Great St. Petersburg Polytechnic University, 195251 St. Petersburg, Russia \\ ${ }^{2}$ Moscow State University of Civil Engineering, Yaroslavskoye shosse, 26, Moscow, 129337, Russia
}

\begin{abstract}
The article considers the compliance of a rigid assembly of a steel column of a typical I-section under the loads of an eccentriccompressive load with a random eccentricity. A simplified scheme of pinching a rigid hinge is considered as well as its detailed version in which the rigid part of the rod is elaborated as a basement, foundation plate, crossarm and anchor bolts with plates. The authors carried out numerical studies of this site using a computer complex Abaqus 2017. Analysis of the results showed that the inclusion of compliance at a sufficiently accurate numerical simulation gives the same value for compressive forces. Thus, this node can be considered rigid, and the coefficient of buckling is almost equal to the theoretical value.
\end{abstract}

\section{Introduction}

Questions of the stability of metal rods have long worried the minds of scientists and engineers. A lot of work has been done to study the behavior of the rod for various fixing, when applying a central or eccentric compressive load, for example, the works of such scientists as F. Bleich, S.P. Tymoshenko. At the moment, design schemes have been developed and the coefficients of the calculated length are derived. However, the question of applying the design scheme to a particular real design is still open and requires detailed study [1].

At present, with the rapid development of technological progress and computer technology, the study of the stability of rods goes to a new level. New computer programs based on the finite element method are capable of processing a large amount of information, thereby obtaining sufficiently accurate models of the behavior of the rods [2].

The aim of the study is to prove the application of the scheme with rigid fixation to the real design of the base of the column, using the software complex «Abaqus» [3]. Also, on the example of this article it is possible to compare the accuracy of calculation with Russian Sets of rules (SP 16.13330) and calculation with software.

This paper demonstrates a comparison of the calculation results for the loss of stability of an extra-iron-compressed steel column of a typical I-cross-section on rigid foundation by means of manual and machine-count rates in the software package [4].

\footnotetext{
*Corresponding author: morozovoff85@gmail.com
} 
The main task of this work is to confirm the equivalence of the operation of the variant of the rigid node of the steel column by means of an analytical calculation and calculation in the Simulia Abaqus 2017 software package. In this paper, the calculation procedure according to the norms of SP 16.13330 for a rod with a hinge on the top while right fixing on the bottom and calculation in a program complex of a flat and three-dimensional model of a similar rod. If the load in three cases is equal, then the simulated 3D node can be considered as hard.

When calculating the strength, it was assumed that the equilibrium of the structure under the action of external forces is stable. However, the failure of the structure can occur due to the fact that the balance of structures due to various reasons will be unstable. In many cases, in addition to checking the strength, it is necessary to check the stability of structural elements. The state of equilibrium is considered stable if, with any possible deviation of the system from the equilibrium position, forces arise that tend to return it to its initial position.

An unstable equilibrium state will be in the case when at least one of the possible deviations of the system from the equilibrium position forces arise, tending to remove it from the initial position.

The equilibrium state will be indifferent if, with different deviations of the system from the equilibrium position, forces arise that tend to return it to its initial position, but at least for one of the possible deviations the system remains in equilibrium in the absence of forces tending to return it to its initial position or to remove from it position.

In the case of loss of stability, the nature of the design work changes, since this type of deformation goes into another, more dangerous, capable of leading it to failure at a load much less than it should be due to strength. It is very important that the loss of stability is accompanied by the growth of large deformations, therefore, this phenomenon has a catastrophic character.

When passing from a stable equilibrium state to an unstable state, the structure passes through a state of indifferent equilibrium. If a slight deviation from the initial position is reported in the state of the structure, then upon termination of the cause that caused this deviation, the structure will not return to its original position, but will be able to retain the new position given to it by the deviation.

The state of indifferent equilibrium, representing, as it were, the boundary between two basic states - stable and unstable, is called a critical state. The load at which the design maintains a state of indifferent equilibrium is called a critical load.

Experiments show that it is usually sufficient to increase the load slightly in comparison with its critical value, so that the structure will lose its bearing capacity due to large deformations, has failed. In the construction industry, the loss of stability by even one element of the structure causes a redistribution of effort throughout the structure and often leads to an accident.

\section{Experimental investigation}

In the calculation, a structural diagram of a column of a typical double-I cross-section fixed rigidly in the lower part and hinged at the top is adopted. At the upper point, a longitudinal compressive load is applied with a random eccentricity equal to $10 \mathrm{~mm}$. For this "rigid-hinge" scheme of the rod, use the coefficient $\mu$, which characterizes the conditions for fixing the compressible element, equal to 0.7 .

The sub-base under the single-footing of the column conditionally accept rigid, as shown in figures 2 and 5, where the base part of the column in the flat examination is fixed rigidly, and in the three - dimensional examination-prohibited the displacement of the foundation part of the column.

The initial data for setting the problem are presented below: 
Table 1. Input data for calculation.

\begin{tabular}{|l|l|l|}
\hline Name & Marking-off & Amount \\
\hline Height of the compressible element & $1, \mathrm{~m}$ & 6 \\
\hline $\begin{array}{l}\text { The cross-sectional area of the compressible } \\
\text { element }\end{array}$ & $\mathrm{A}, \mathrm{cm}^{2}$ & 110.8 \\
\hline The moment resistance & $\mathrm{W}, \mathrm{cm}^{3}$ & 1265 \\
\hline The inertia couple & $\mathrm{I}, \mathrm{cm}^{4}$ & 18849 \\
\hline $\begin{array}{l}\text { The radius of inertia of the section of the } \\
\text { compressible element }\end{array}$ & $\mathrm{i}, \mathrm{cm}$ & 13.04 \\
\hline Mechanical properties of the material of the compressible element & 230 \\
\hline $\begin{array}{l}\text { The elasticity limit of the material of the } \\
\text { compressible element }\end{array}$ & $\mathrm{R}, \mathrm{MPa}$ & 206 \\
\hline $\begin{array}{l}\text { The modulus of elasticity of the material of the } \\
\text { compressible element }\end{array}$ & $\mathrm{E}, \mathrm{GPa}$ & \\
\hline
\end{tabular}

The calculated effective rod length of the compressible element is given by the formula:

$$
l_{e f}=\mu \cdot l \text {, }
$$

where, 1 - the geometric length of the element;

$\mu$ - coefficient, depending on the method of fixing the rod.

This rod is shown in Fig. 1.

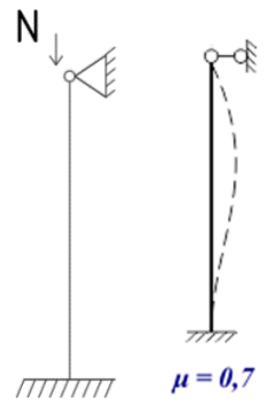

Fig. 1 The calculated scheme of a compressed rod and its deformed scheme.

Flexibility of the element:

$$
\lambda=\frac{l_{e f}}{i} .
$$

Define ...

$$
m=\frac{e \cdot A}{W}
$$

where $\mathrm{m}$ - imperfection.

We determine the conditional flexibility of the element:

$$
\lambda_{y}=\lambda \cdot \sqrt{\frac{R_{y}}{E}} .
$$

From the table from the SP we take the coefficient of longitudinal bending. $\varphi_{e}=0.9$ [5]. The ultimate axial force at the time of loss of stability [5]: 


$$
N_{u l t}=\varphi_{e} A R_{y} \gamma_{c}
$$

where $\gamma_{\mathrm{c}}$-specific-conditions-of-use factor, equal to 1 .

Using these formulas, we calculate:

$$
\begin{gathered}
l_{e f}=0.7 \cdot 6=4.2 \mathrm{~m} . \\
\lambda=\frac{4,2}{13.04}=32.21 . \\
m=\frac{1 \cdot 110.8}{1265}=0.088 . \\
\lambda_{y}=32.21 \cdot \sqrt{\frac{230}{2.06 \cdot 10^{5}}}=1.076 . \\
\varphi_{e}=0.9 . \\
N_{u l t}=0.9 \cdot 110.8 \cdot 230=2294 \mathrm{kN} .
\end{gathered}
$$

The modeling of the rod and the task of external loading in the ABAQUS CAE software is shown in Figure 2, where the method of pinching the rod at the top and bottom is given conventionally with the appropriate notation [6-7].

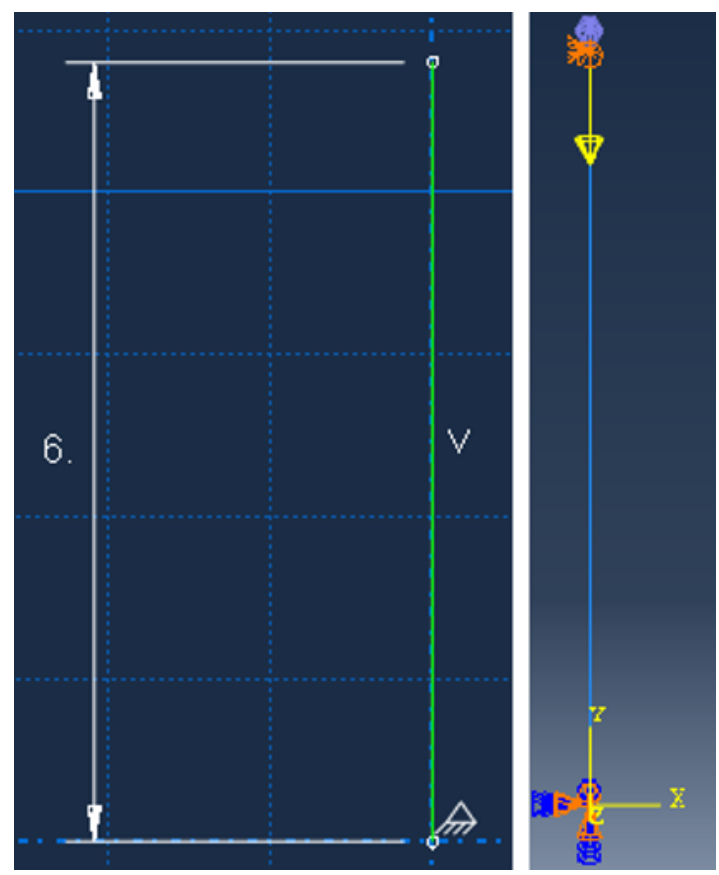

Fig. 2 Modeling the rod.

The result of the calculation of the program is shown in Fig. 3. 


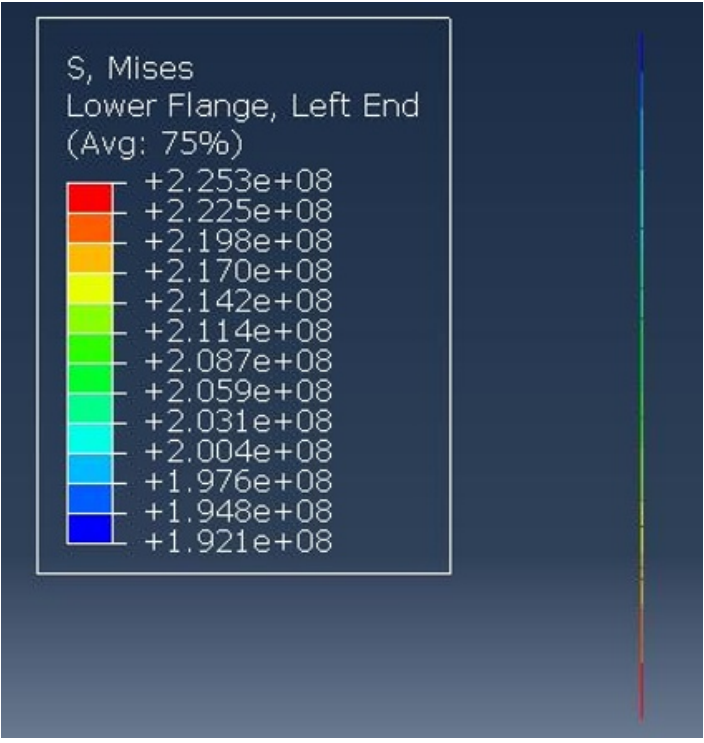

Fig. 3 Visual calculation result.

Imagine it in the form of a graph, which is depicted in Fig. 4.
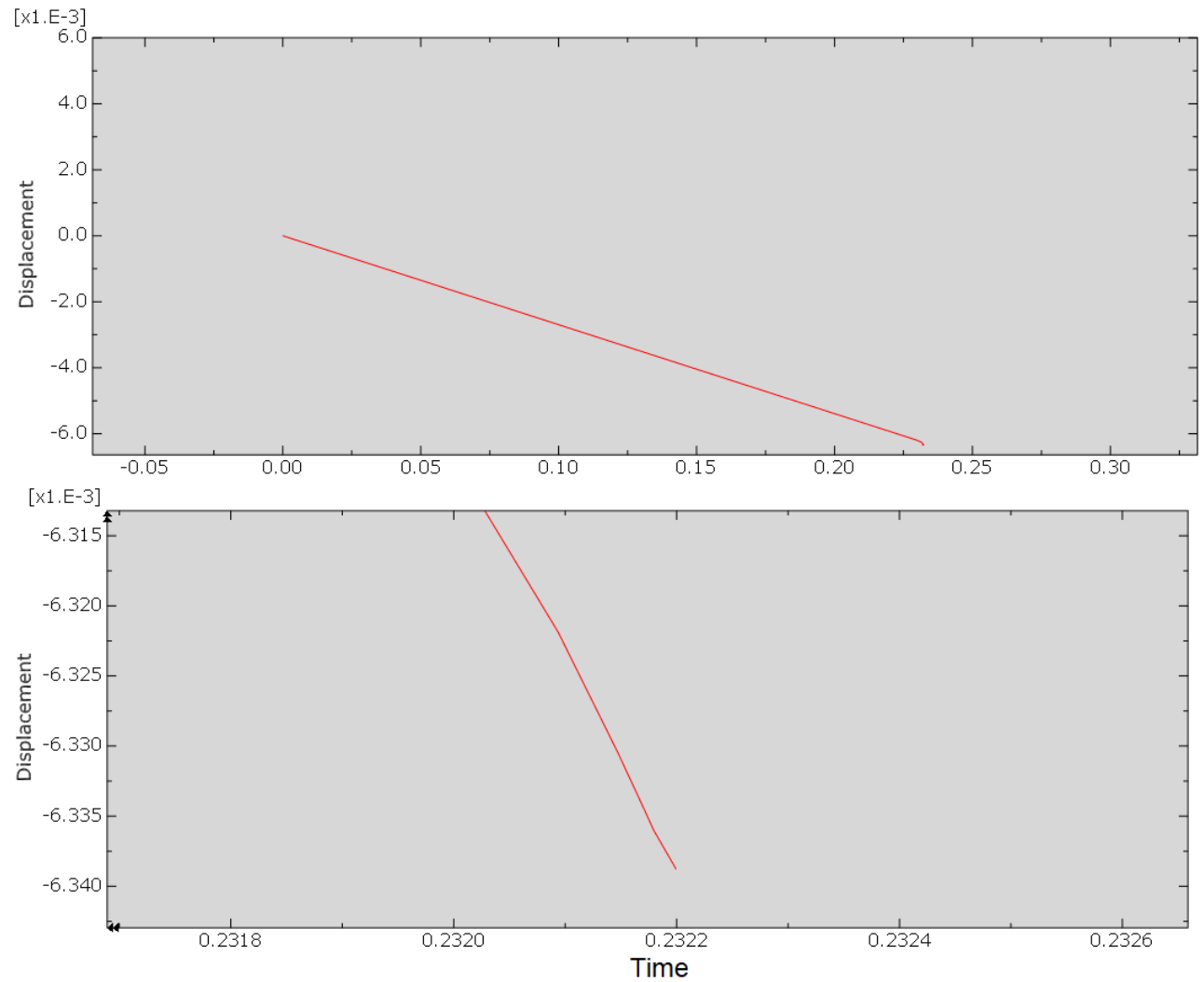

Fig. 4 Result of loss of stability of the rod. 
In this way, the calculation showed that the rod loses stability under an imperfectionally applied load equal to $2322 \mathrm{kN}$. Abscissa units are increments of loading steps. The linear calculation consists of one step is loading.

This model is performed in a similar way, except for the rigid fixing, the area of which is cut to $0.5 \mathrm{~m}$ and is modeled as a foundation, foundation plate, wing plates, steel plate and anchors.
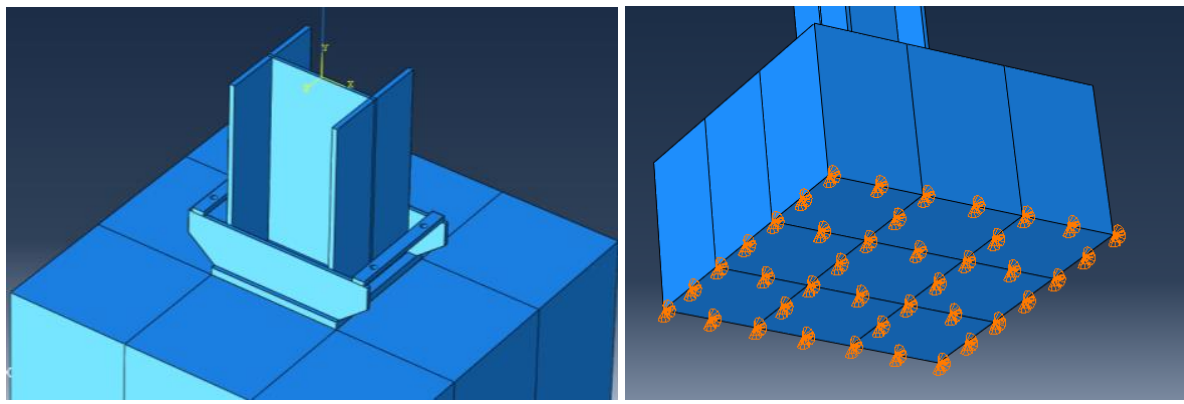

Fig. 5 The model of the rigid fixing of the column rod on rigid foundation.

Similarly, a load is applied with an imperfection load. The result of the calculation is shown graphically in Fig. 6.

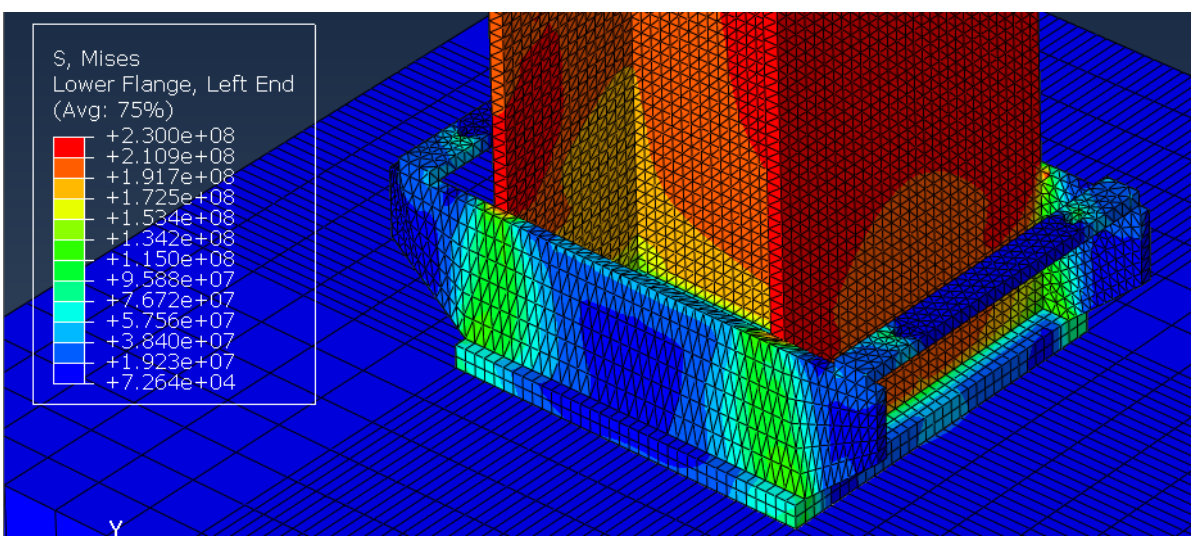

Fig. 6 Visual result of calculating the column rod (Mises stresses).

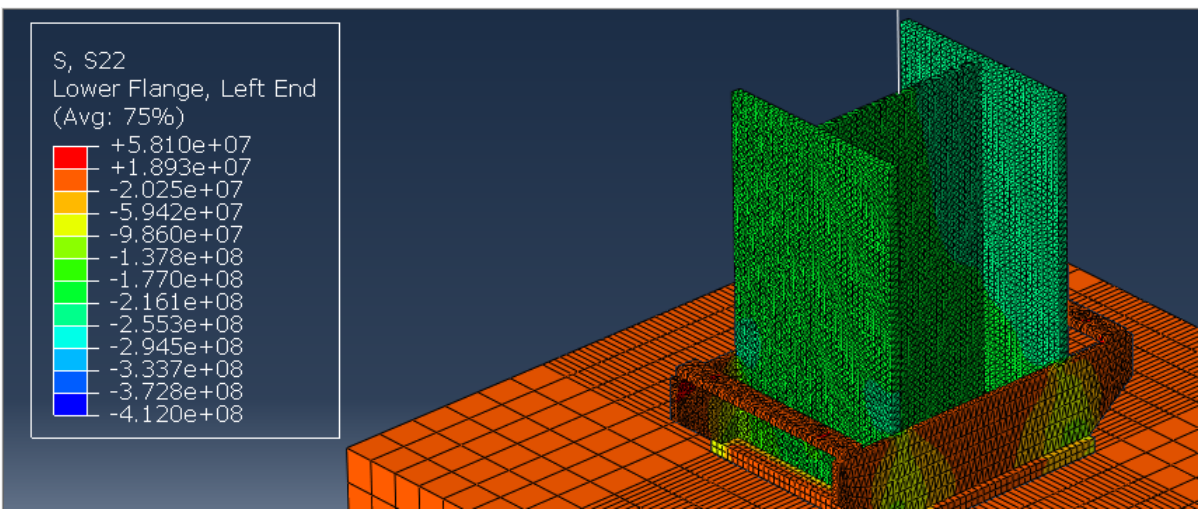

Fig. 7 Visual result of calculating the column rod (Y-axis stresses).

The obtained results are presented in the form of a graph. It is shown in Fig. 8. 

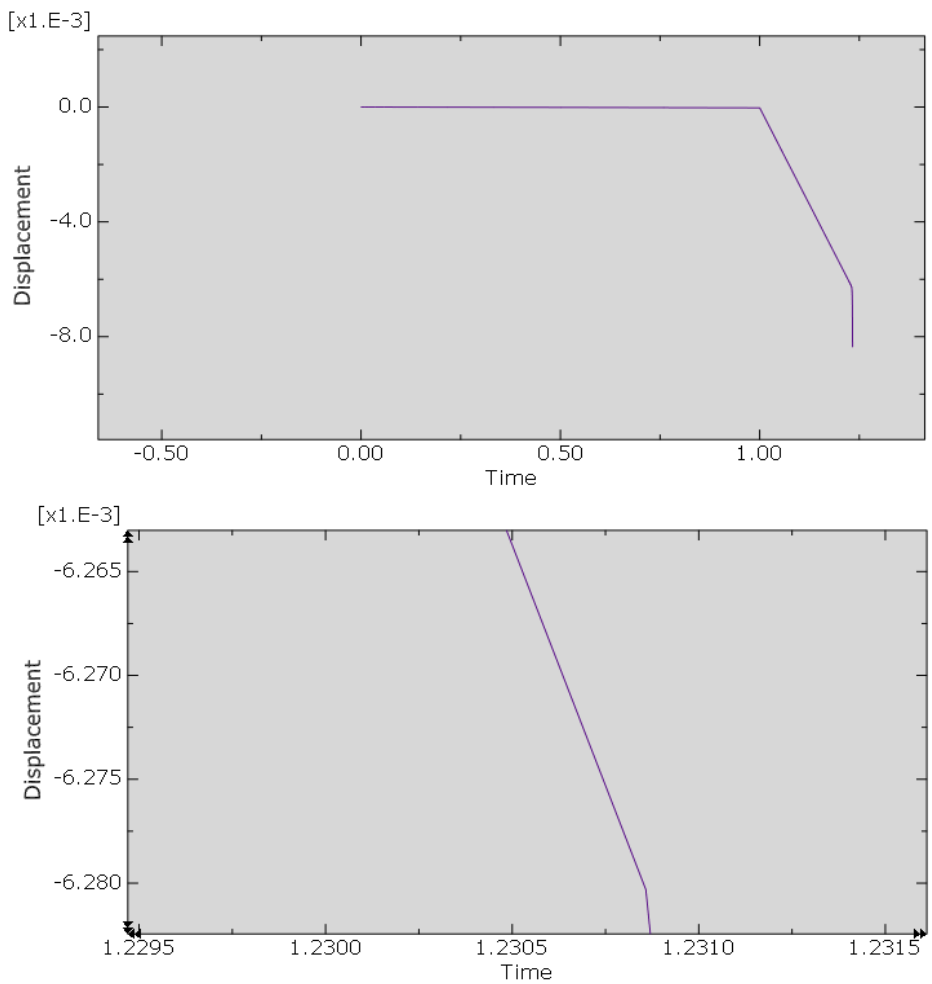

Fig. 8 The result of loss of stability of the rod with a simulated rigid node.

Thus, the calculation showed that the rod loses stability under an imperfection applied load equal to $2309 \mathrm{kN}$. Here are two steps of calculation: the first is pre-loading, the second is loading.

\section{Results and Discussion}

As a result, the reliability of the results is confirmed by their approximate equality with three methods of calculation, as can be seen in Fig. 9.

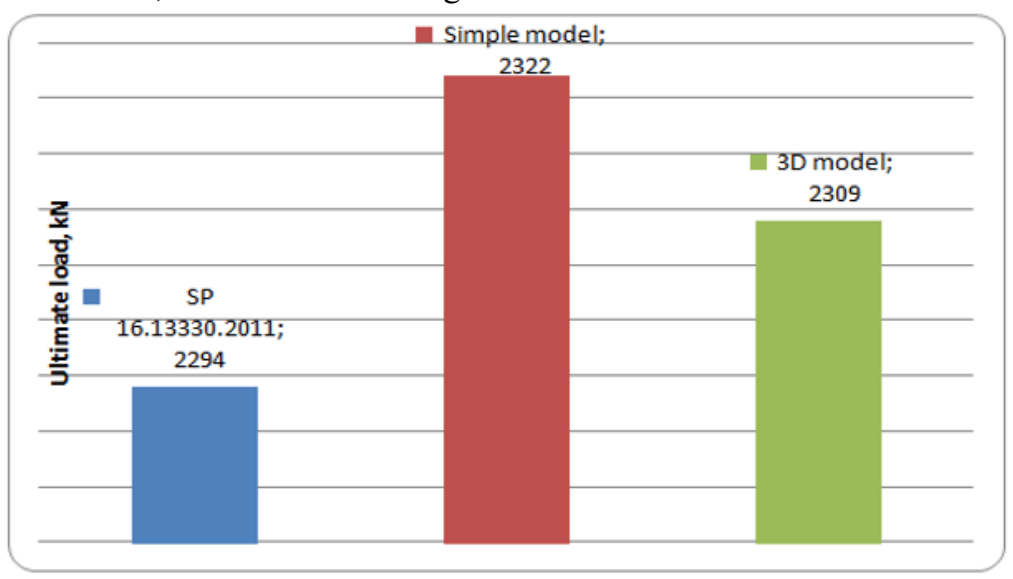

Fig. 9 Comparison of calculation results 


\section{Conclusions}

1. The results of the research allow us to say that the design scheme of rigid-hinged fastening is analogous to the actual construction of the base of the column on rigid foundation, which can be considered rigidly fixed.

2. The difference in the calculation results is extremely small, which allows us to neglect it.

3. Also, the results of this work allow us to conclude that to this core of a steel column of a typical I-beam profile it is possible to apply a load with an imperfection greater than the calculation in the software package than calculated analytically $15 \mathrm{kN}$ or 1.5 tons, which is $0.65 \%$ :

$$
\Delta=\frac{2309-2294}{2309} * 100 \%=0.65 \% \text {. }
$$

\section{References}

1. V.V. Lalin, L.A. Rozin, Mag. Civ. Eng., 87-96, 36, 1 (2013) doi: 10.5862/MCE.56.7

2. O.A., Sergeyev, Mag. Civ. Eng., 9, 44, (2013) 10.5862/MCE.44.4

3. H. Ziegler, Principles of Structural Stability. (Springer Basel AG. Stuttgart, 1977)

4. E. Riks,. Adv. Appl. Mech, 34 (34), (1998) doi: 10.1016/S0065-2156(08)70319-3

5. Z.P. Bazant, L. Cedolin, Oxford University Press. Oxford, (1991).

6. Vatin N., Bagautdinov R., Andreev K., Applied Mechanics and Materials, 725-726, (2015) doi.org/10.4028/www.scientific.net/AMM.725-726.710

7. F. Ragheb Wael, Thin-Walled Structures, 97, (2015) 